\title{
PP18
}

\section{EFFECT OF INTENSITY OF SHORT-WAVELENGTH LIGHT ON SUBJECTIVE AND OBJECTIVE ALERTNESS}

\author{
Jing Lin et al. \\ DOI 10.25039/x46.2019.PP18 \\ from \\ CIE x046:2019 \\ Proceedings \\ of the \\ 29th CIE SESSION \\ Washington D.C., USA, June 14 - 22, 2019 \\ (DOI 10.25039/x46.2019)
}

The paper has been presented at the 29th CIE Session, Washington D.C., USA, June 14-22, 2019. It has not been peer-reviewed by CIE.

(C) CIE 2019

All rights reserved. Unless otherwise specified, no part of this publication may be reproduced or utilized in any form or by any means, electronic or mechanical, including photocopying and microfilm, without permission in writing from CIE Central Bureau at the address below. Any mention of organizations or products does not imply endorsement by the CIE.

This paper is made available open access for individual use. However, in all other cases all rights are reserved unless explicit permission is sought from and given by the CIE.

CIE Central Bureau

Babenbergerstrasse 9

A-1010 Vienna

Austria

Tel.: +4317143187

e-mail: ciecb@cie.co.at

www.cie.co.at 


\title{
EFFECT OF INTENSITY OF SHORT-WAVELENGTH LIGHT ON SUBJECTIVE AND OBJECTIVE ALERTNESS
}

\author{
Lin, J., Westland, S., \\ University of Leeds, Leeds, UNITED KINGDOM \\ cm14jl@leeds.ac.uk
}

DOI 10.25039/x46.2019.PP18

\begin{abstract}
Short-wavelength light has been known to have an alerting effect on human alertness in the night-time. However, there is very few study focus on the effect of intensity of light on alertness. In this study we evaluated alerting ability of short-wavelength light of three different intensities (40lx, 80lx and 160lx). Eight subjects participated in a 60-minute exposure protocol for four evenings, during which electroencephalogram (EEG) as well as subjective sleepiness was collected. EEG power in the beta range was significantly higher after subjects were exposed to $1601 x$ light than after they were exposed to $401 x, 801 x$ light or remained in darkness. Also, the alpha theta was significantly lower under $160 \mathrm{~lx}$ light then in darkness. These results showed that the effect of intensity on alertness is not linear and further work should be done to investigate the threshold intensity that is required to produce alerting effect.
\end{abstract}

Keywords: Alertness, Lighting, EEG

\section{Introduction}

It is now well known that exposure to light in the evening and night-time, especially of shortwavelength (but not necessarily limited to short wavelengths), leads to an increase in alertness in humans. There is evidence that exposure to light at night promotes alertness in humans and it has been suggested that this effect is related to circadian disruption. Circadian disruption is associated with reduced levels of the hormone melatonin and is primarily mediated by activation of the intrinsically photosensitive Retinal Ganglion Cells (ipRGCs) which respond to shortwavelength light most strongly.

It has been shown that exposure to light in the evening can inhibit the production of melatonin which otherwise would naturally build-up in the body during the late-evening hours. Studies to date have linked the alerting effects of light to its ability to suppress melatonin (Figueiro, 2007). However, some other studies have also shown that acute melatonin suppression is not needed for light to affect alertness at night. It has also been demonstrated that both blue and red lights increase beta power and reduced alpha theta power relative to darkness, though only blue light suppressed melatonin significantly (Figueiro, 2009). These findings suggest that although melatonin level is associated with circadian clock and sleep, electroencephalogram (EEG) measures might reflect instant neuroendocrine responses and objective alertness.

Previous studies have used many measures in order to evaluate alertness. Subjective sleepiness is often rated using Karolinska Sleepiness Scale (KSS), a self-reported scale that ranges from 1 ('very alert') to 9 ('very sleepy, fighting sleep'). The KSS measures situational sleepiness and it is sensitive to fluctuations (Shahid, 2011). As a more quantitative measure, EEG has also been commonly used in the assessment of objective sleepiness. Research explored the correlation between EEG power spectrum and alertness (Jung, 1997).

It has been shown that EEG power in certain frequency ranges changes significantly as the status of the subject changes. One study investigated how 48-minute exposures to three lighting conditions (red, blue and dark) affected EEG measures in subjects; it was found that alpha and alpha theta power were both lower after the exposure to red and blue lights compared to darkness (Sahin, 2013). Another study also compared short wavelength light, long wavelength light and darkness, and found that alpha power was significantly lower after 30 minutes under both the short- and long-wavelength light than remaining in darkness (Okamoto, 2014). Red 
and blue lights have been found to increase beta, reduced sleepiness relative to preceding dim light exposure, although these did not reach statistical significance (Plitnick, 2010). By looking into individual EEG frequencies, measuring melatonin level and conducting a vigilance test, it was suggested that short-wavelength light enhanced high alpha activity (Lockley, 2006), which is seen as a specific marker of the endogenous circadian drive for alertness (Aeschbach, 1999). It has also been shown that light can have an impact on EEG measures without affecting melatonin levels (Figueiro, 2016). As we see, the impact of light on alertness is a complex physical, physiological and psychological activity that can be resulted from different pathways, where through hormonal melatonin is one of them. EEG, however, reflects a more instant alertness fluctuation.

Short-wavelength light is becoming a critical safety concern. For example, there has been concern about home lighting in the evening, where warm Tungsten light is increasingly being replaced by cooler solid-state lighting; the use of emissive electronic displays may also be responsible for the increasing sleep problem (Chang, 2015). However, there is no consensus yet about the threshold of light intensity required to produce these effects. It is likely that the threshold will depend on the wavelength distribution of the light, how long the exposure is for, and the time of day that the exposure takes place; this may explain in part why it has so far proven difficult to measure the threshold. A study has looked at how three different illuminances ranging from 3 to $91001 x$ affect EEG measure over 6.5 hours of exposure, and a dose-response relationship was found in subjective alertness and theta alpha power (Cajochen, 2000). Many other such studies have demonstrated a non-linear relationship between light intensity and circadian shifts (Boivin, 1996; Zeitzer, 2000). It is generally agreed that brighter light has a stronger alerting ability than dimmer light. The question remains, however, whether there is a threshold and what that threshold is? Crucially, is the light from electronic devices we constantly use at night bright enough to trigger these effects?

Very few studies have looked into the effect of intensity of short wavelength light. Blue light of 40 Ix has been investigated in some studies (Sahin, 2013; Okamoto, 2014), and an alerting effect has been suggested. Some other studies, for example, have looked at light of $401 \mathrm{x}$ at $2500 \mathrm{~K}, 3000 \mathrm{~K}$ and $6500 \mathrm{~K}$ (Chellappa, 2011), and light of 295Ix at $2700 \mathrm{~K}$ and $209 \mathrm{Ix}$ at $5600 K$ (Nagare, 2018). These studies, however, focussed on spectral composition or exposure duration, rather than light intensity.

This work is concerned with measuring the threshold intensity of light needed to increase alertness and uses both subjective (KSS) and objective (EEG) methods, based on the notion that melatonin is not the only mechanism contributing to light-induced alertness. In this study, specifically, the objective is to investigate the effect of three intensities (40lx, 80lx and 160lx) of a blue light $(\lambda \max =470 \mathrm{~nm})$, compared to darkness $(<1 \mathrm{~lx})$, on human alertness during the evening.

\section{Experimental}

\subsection{Subjects}

Nine participants were recruited and all of them went through a pre-screening procedure using an adapted version of Munich ChronoType Questionnaire (Roenneberg, 2003). Individual sleep/rise time was collected and the daily consumption of nicotine, caffeine and alcohol was reported. Smokers and those who were rated as extreme late/early chronotypes were excluded. The study was approved by the University of Leeds Ethics Committee and all subjects signed informed consent. An information sheet was given and participants were asked to refrain from caffeine and alcohol intake 3 hours prior to the experiment, and to try to maintain a regular, constant sleep schedule during the entire experimental period. As a result, there is no report of any major health problems or colour blindness. One participant did not finish all experimental sessions, and their data was excluded from further analysis. In total, the result produced from eight subjects (five females, mean age 28 ) is reported here.

\subsection{Lighting conditions}

Light was delivered through 12 luminaires (LEDs, provided by Thouslight Lighting System) mounted in the ceiling of a room with white walls and grey carpets. Participants were asked to sit inside the room whilst reading, with a white table in front of them (Figure 1). 


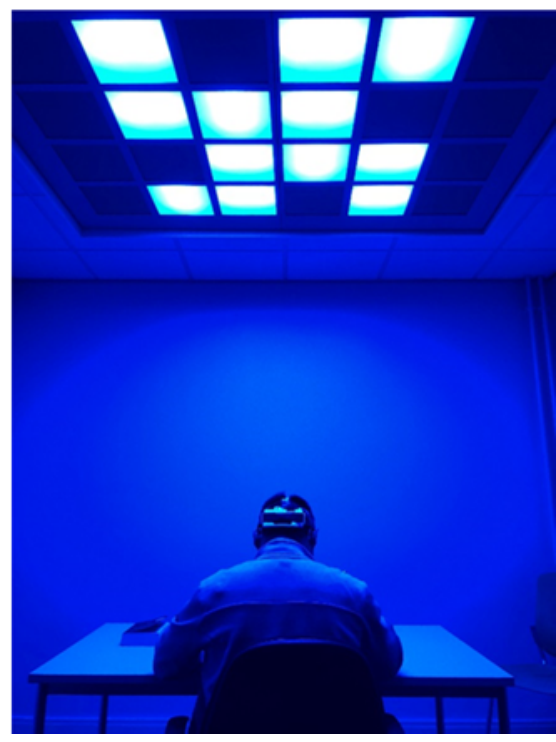

Figure 1 - Lighting Room showing the position of the subject and the luminaires

Four light settings were used: a dark control $(2000 \mathrm{~K},<1 \mathrm{~lx}$ at eye level) and three experimental lighting conditions). The short wavelength light had a peak at about $470 \mathrm{~nm}$ and was approximately Gaussian with a half-width half-height of $35 \mathrm{~nm}$. Three intensities were 40,80 and I60Ix at eye level. The spectra of the three test lights were measured with an Xrite i1 Display pro (see Figure 2).

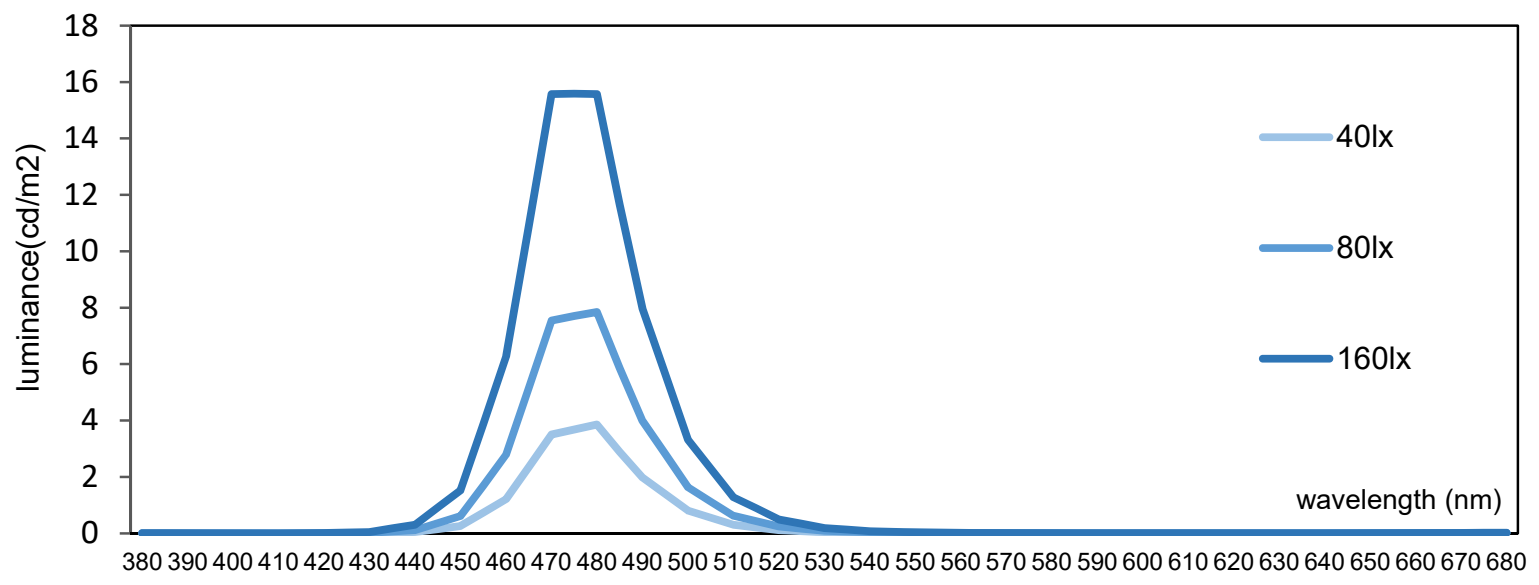

Figure 2 - Spectra of three test lighting conditions

\subsection{Experiment protocol}

Each subject completed four sessions over four nights, all starting at the same time (8pm). Participants were fitted with EEG electrodes prior to the start of the exposure. The order of the conditions (dark, 40Ix, 80Ix and 160lx) was selected randomly for each of the participants to avoid potential sequence effects. During each evening study EEG was continuously recorded over 60 minutes (20 minutes for dark adaption and 40 minutes for the blue light) and subjective sleepiness (which was assessed using the KSS) was rated every 20 minutes (three times in total). For the duration of the 60 minutes the participants were free to read a book. They were also asked to keep their eyes open and reduce head movement throughout the experiment. No other activities (e.g. using electronic devices, eating or talking) were allowed (Figure 3). 


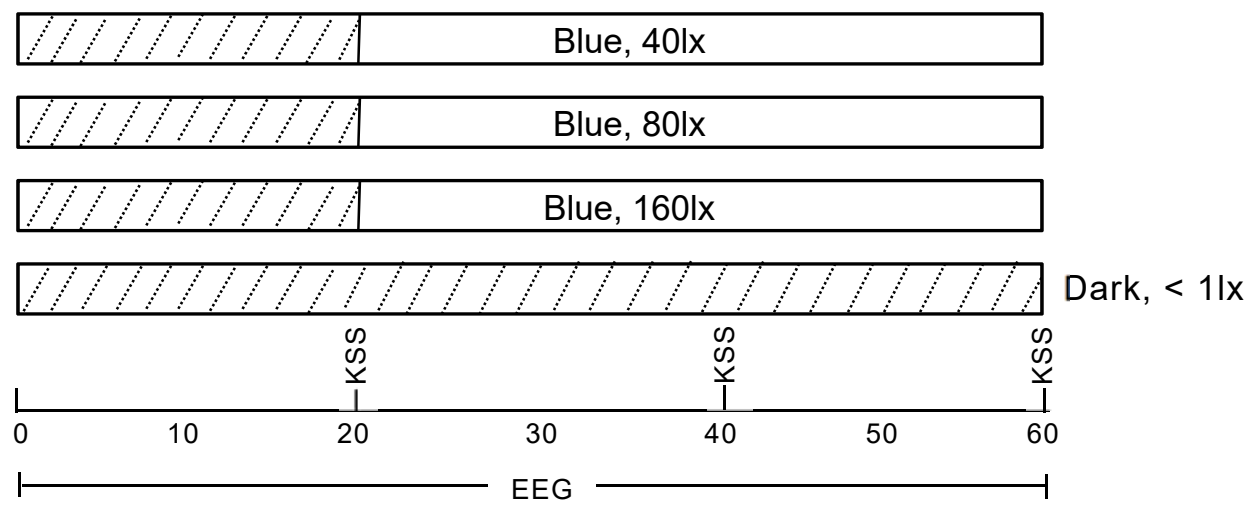

Elapsed Time (min)

Figure 3 - Experimental design

\section{Results}

\subsection{EEG}

EEG data was collected using B-Alert Live Software (BLS) with wireless ABM EEG device (X10 headset with standard sensor strips). Recordings consisted of EEG with 9 electrode positions ( Fz, Cz, Poz, F3, F4, C3, C4, P3, and P4) and two reference mastoid electrodes. The electrode impedance test was performed each time before experiment to ensure the good conductivity between the scalp and electrodes, thus the good quality of the signal. The EEG signal was band-passed to 1 to $40 \mathrm{~Hz}$ and decontaminated using ABM's validated artifact identification and decontamination algorithms which identify and remove 5 artifact types: EMG, EOG, excursions, saturations, and spikes. Power spectral density (PSD) was computed by performing Fast Fourier Transform (FFT) with application of a Kaiser window. PSD of selected 1- $\mathrm{Hz}$ bins was averaged after application of a 50\% overlapping window across three one-second overlays.

EEG measures collected from 9 electrode sites were averaged to produce overall EEG power, and was then grouped into the following frequency bins: $5-9 \mathrm{~Hz}$ (theta alpha), 8-9 Hz (lower alpha), $11-13 \mathrm{~Hz}$ (higher alpha), and $13-30 \mathrm{~Hz}$ (beta). In each frequency range, EEG power averaged over the 40 minutes under experimental lighting was normalized to the initial 20 minutes of dark adaption. One-way analysis of variance (ANOVA) was performed using the normalized power in each of the frequency ranges studied. Post-hoc (with LSD) was used to further compare the significance between lighting conditions. Analyses were performed using IBM SPSS Statistics 25 and the results for theta alpha and beta ranges are listed in Table 1.

\section{Table 1 - Pairwise comparisons for EEG theta alpha and beta power}

\begin{tabular}{|c|c|c|}
\hline & Theta alpha & Beta \\
\hline Pairs & \multicolumn{2}{|c|}{ Sig. $^{*}$} \\
\hline Dark-40Ix & $0.037^{*}$ & 0.508 \\
\hline Dark-80lx & 0.189 & 0.214 \\
\hline Dark-160lx & $0.012^{*}$ & $0.000^{*}$ \\
\hline 401x-80Ix & 0.407 & 0.553 \\
\hline 401x-160lx & 0.610 & $0.001^{*}$ \\
\hline 801x-160lx & 0.186 & $0.004^{*}$ \\
\hline \multicolumn{2}{|c|}{${ }^{*}$ Statistically significant $(p \leq 0.05)}$. \\
\hline
\end{tabular}

One-way ANOVA revealed a significant main effect of lighting conditions in the normalized theta alpha and beta ranges. Significant differences are found between: dark - 40lx and dark - 160lx in theta alpha; dark -160lx, 40lx - 160lx and 80lx - 160lx in beta. Significant difference was not observed in lower alpha or high alpha range. Figure 4 shows the results of normalized power 
for four lighting conditions in four frequency ranges studied (where *indicates significance). Power in theta alpha was significantly lower after exposure to $401 x$ and $1601 x$ blue lights than after remaining in the dark condition. Power in beta range was significantly higher after exposure to $160 \mathrm{~lx}$ blue lights than after exposure to the other three lighting conditions. Compared to dark, exposure to $160 \mathrm{~lx}$ blue light has also reduced lower alpha power and increased high alpha power, although these differences did not reach statistical significance $(p>0.05)$.

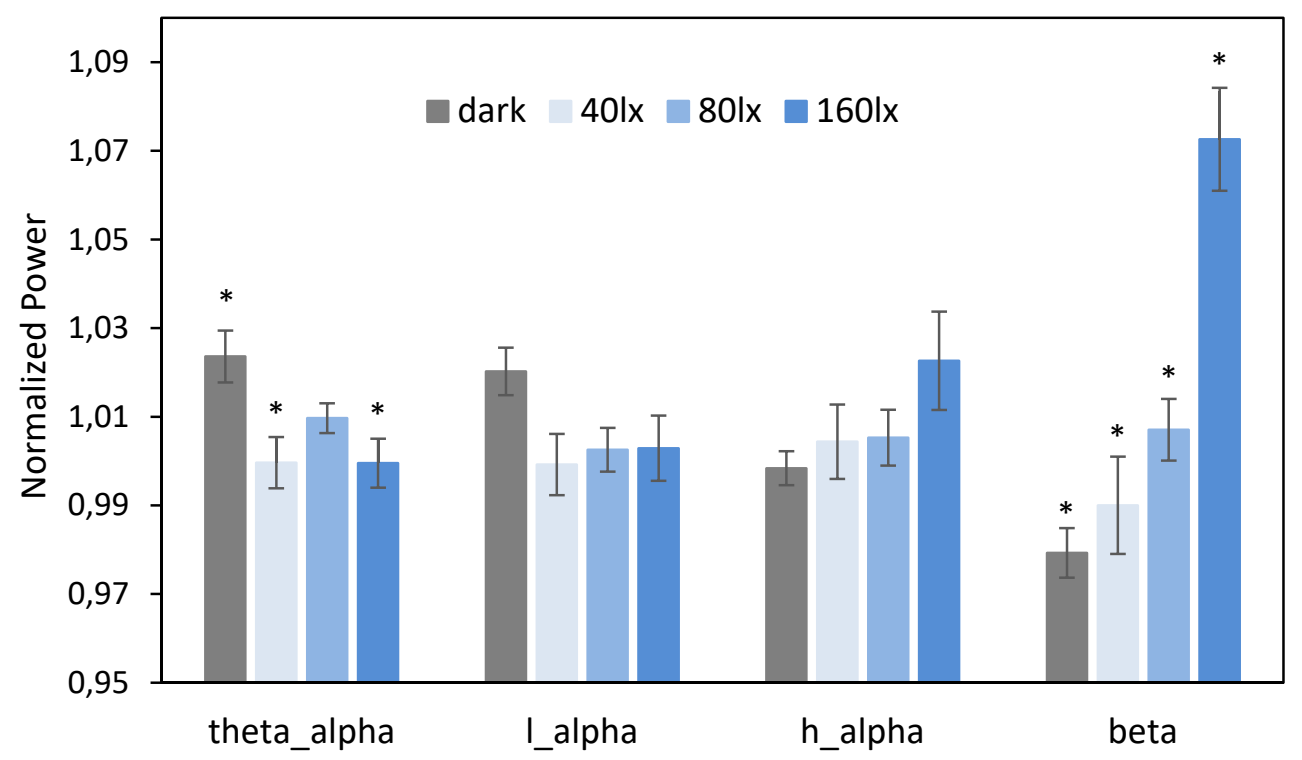

Figure 4 - Average \pm standard error of the mean normalized EEG power for four frequency ranges

\subsection{Subjective sleepiness (KSS)}

Subjective sleepiness was rated three times (at the $20 \mathrm{~min}, 40 \mathrm{~min}$, and at the end) using KSS. Subjects were asked to rate themselves as ranged from 1 (extremely alert) to 9 (very sleepy, great effort to keep awake, fighting sleep). Mean score over the experimental condition was normalized to the initial dark adaption. One-way ANOVA was performed and post-hoc (with LSD) was used to further compare the significance between lighting conditions. The results are listed in Table 2.

\section{Table 2 - Pairwise comparisons for KSS scores}

\begin{tabular}{|c|c|}
\hline Pairs & Sig. $^{*}$ \\
\hline Dark-40Ix & 0.240 \\
\hline Dark-80Ix & 0.252 \\
\hline Dark-160lx & $0.024^{*}$ \\
\hline $401 x-801 x$ & 0.973 \\
\hline $401 x-1601 x$ & 0.209 \\
\hline 80lx-160lx & 0.198 \\
\hline " Statistically significant ( $\mathrm{p} \leq 0.05)$.
\end{tabular}

ANOVA showed a significant difference between dark and 160lx conditions. Figure 5 shows the results for the normalized KSS scores under four lighting conditions (where *indicates significance). Mean score in $1601 x$ condition was significantly lower than score in dark condition (a lower KSS score means more alertness). Mean scores under $40 \mathrm{Ix}$ and $80 \mathrm{Ix}$ conditions were lower than score under the dark, and higher than score under the $1601 x$ condition, although these differences did not reach statistical significance $(p>0.05)$. 


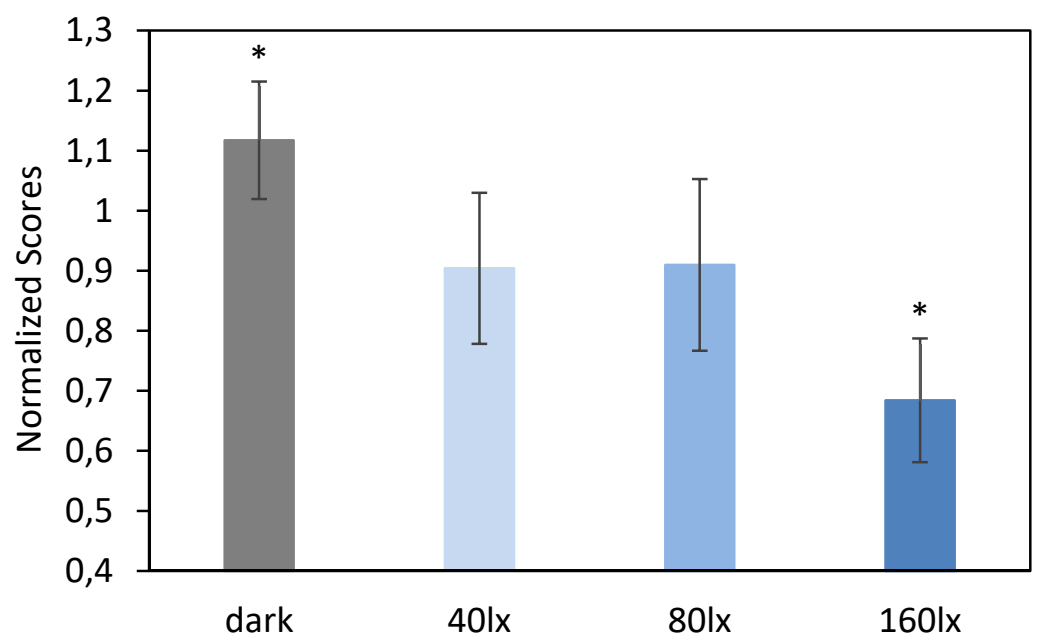

Figure 5 - Average \pm standard error of the mean normalized KSS scores (lower scores indicate greater alertness)

\section{Discussion}

The present study investigated how exposures to short wavelength lights of three different intensities (40lx, 80lx and 160lx) affect objective and subjective alertness during the night time. EEG showed that intensity has a significant effect on theta alpha $(5-9 \mathrm{~Hz})$ and beta $(13-30 \mathrm{~Hz})$ power. Exposure to $40 \mathrm{~lx}$ and $160 \mathrm{Ix}$ lights significantly reduced theta alpha power compared to dark. Exposure to $160 \mathrm{~lx}$ light significantly increased beta power compared to dark, $401 \mathrm{x}$ and $80 \mathrm{~lx}$ lights. It has been observed and agreed in many studies that the reduction in theta alpha and the increase in beta indicate greater alertness. The results of this study showed that exposure to $160 \mathrm{~lx}$ blue light significantly increases alertness compared to dark, $40 \mathrm{~lx}$ and $80 \mathrm{~lx}$; exposure to $40 \mathrm{~lx}$ and $80 \mathrm{~lx}$ might also increase alertness compared to dark, although not as significant as $1601 x$ light. Furthermore, it did not show a large difference between 40 and $801 x$ exposure.

Subjective alertness (KSS) reported is consistent with EEG results. Subjects rated themselves as sleepier in the dark, and more alert under the blue lights. With the dark condition having the highest score and 160lx having the lowest, this again shows a significant alerting effect of $160 \mathrm{~lx}$ compared to dark. $401 x$ and $801 x$ exposure were rated in between the dark and $1601 x$ conditions, at about the same level.

This study extends the research on the effect of intensity on light-induced alertness. The findings suggested that, firstly, a certain level of intensity is needed to induce alertness (to produce a significant effect a higher level of intensity might be needed). Secondly, the relations between intensity and alertness change is not linear, which certain parameters e.g. threshold intensity that could maximum increase alertness might be identified.

\section{Conclusions}

The present study provides some evidence that short-wavelength light exposure in the evening can increase human alertness and that this can occur relatively quickly (even though some other studies have suggestion that melatonin inhibition, for example, may have a longer time course). Both objective and subjective results also suggest that for the lighting conditions tested in the present study, light of higher intensity has a stronger alerting effect than light of lower intensity. These findings, in themselves, do not enable a threshold effect to be identified. However, the methodology described in this study may provide a basis for future on-going work to address this question explicitly. 


\section{References}

FIGUEIRO, M.G., BULLOUGH, J.D., BIERMAN, A., FAY, C.R. and REA, M.S., 2007. On light as an alerting stimulus at night. Acta neurobiologiae experimentalis, 67(2), 171.

FIGUEIRO, M.G., BIERMAN, A., PLITNICK, B. and REA, M.S., 2009. Preliminary evidence that both blue and red light can induce alertness at night. BMC neuroscience, 10(1), 105.

SHAHID, A., WILKINSON, K., MARCU, S. and SHAPIRO, C.M., 2011. Karolinska sleepiness scale (KSS). STOP, THAT and One Hundred Other Sleep Scales, 209-210. New York: Springer.

JUNG, T.P., MAKEIG, S., STENSMO, M. and SEJNOWSKI, T.J., 1997. Estimating alertness from the EEG power spectrum. IEEE transactions on biomedical engineering, 44(1), 60-69.

SAHIN, L. and FIGUEIRO, M.G., 2013. Alerting effects of short-wavelength (blue) and longwavelength (red) lights in the afternoon. Physiology \& behavior, 116, 1-7.

OKAMOTO, Y., REA, M.S. and FIGUEIRO, M.G., 2014. Temporal dynamics of EEG activity during short-and long-wavelength light exposures in the early morning. BMC research notes, 7(1), 113.

PLITNICK, B., FIGUEIRO, M.G., WOOD, B. and REA, M.S., 2010. The effects of red and blue light on alertness and mood at night. Lighting Research \& Technology, 42(4), 449-458.

LOCKLEY, S.W., EVANS, E.E., SCHEER, F.A., BRAINARD, G.C., CZEISLER, C.A. and AESCHBACH, D., 2006. Short-wavelength sensitivity for the direct effects of light on alertness, vigilance, and the waking electroencephalogram in humans. Sleep, 29(2), 161168.

AESCHBACH, D., MATTHEWS, J.R., POSTOLACHE, T.T., JACKSON, M.A., GIESEN, H.A. And WEHR, T.A., 1999. Two circadian rhythms in the human electroencephalogram during wakefulness. American Journal of Physiology-Regulatory, Integrative and Comparative Physiology, 277(6), R1771-R1779.

FIGUEIRO, M.G., SAHIN, L., WOOD, B. and PLITNICK, B., 2016. Light at night and measures of alertness and performance: implications for shift workers. Biological research for nursing, 18(1), 90-100.

CHANG, A.M., AESCHBACH, D., DUFFY, J.F. and CZEISLER, C.A., 2015. Evening use of lightemitting eReaders negatively affects sleep, circadian timing, and next-morning alertness. Proceedings of the National Academy of Sciences, 112(4), 1232-1237.

CAJOCHEN, C., ZEITZER, J.M., CZEISLER, C.A. and DIJK, D.J., 2000. Dose-response relationship for light intensity and ocular and electroencephalographic correlates of human alertness. Behavioural brain research, 115(1), 75-83.

BOIVIN, D.B., DUFFY, J.F., KRONAUER, R.E. and CZEISLER, C.A., 1996. Dose-response relationships for resetting of human circadian clock by light. Nature, 379(6565), 540.

ZEITZER, J.M., DIJK, D.J., KRONAUER, R.E., BROWN, E.N. and CZEISLER, C.A., 2000. Sensitivity of the human circadian pacemaker to nocturnal light: melatonin phase resetting and suppression. The Journal of physiology, 526(3), 695-702.

CHELLAPPA, S.L., STEINER, R., BLATTNER, P., OELHAFEN, P., GÖTZ, T. and CAJOCHEN, C., 2011. Non-visual effects of light on melatonin, alertness and cognitive performance: can blue-enriched light keep us alert?. PloS one, 6(1), e16429.

NAGARE, R., PLITNICK, B. and FIGUEIRO, M.G., 2018. Effect of exposure duration and light spectra on nighttime melatonin suppression in adolescents and adults. Lighting Research \& Technology, 1477153518763003.

ROENNEBERG, T., WIRZ-JUSTICE, A. and MERROW, M., 2003. Life between clocks: daily temporal patterns of human chronotypes. Journal of biological rhythms, 18(1), 80-90. 